\title{
Las subespecies de Lonchocarpus rugosus Benth. (Leguminosae, Papilionoideae: Millettieae)
}

\section{Mario Sousa S. ${ }^{1}$}

Resumen. Se consideran a cuatro subespecies para Lonchocarpus rugosus Benth., de ellas una es nueva para la ciencia y en otra se realiza una nueva combinación, todas se describen y se ilustran dos, la nueva para la ciencia y la combinación propuesta.

Palabras clave: Centroamérica, leguminosa, México.

Abstract. Four subspecies are included in Lonchocarpus rugosus Benth., a new one is described for science and also a new combination is done. All are described and the new ones are also illustrated.

Key words: Central America, legume, Mexico.

\section{Introducción}

En la elaboración del tratamiento del género Lonchocarpus Kunth (Leguminosae, Papilionoideae: Millettieae) para la Flora Mesoamericana, Lonchocarpus rugosus Benth. ha resultado ser una de las especies de más amplia distribución y variabilidad en el género. Hermann (1948) abordo a esta especie ampliando su concepto al incluir a L. apricus Lundell, L. gillyi Lundell, L. hidalgensis Lundell y L. hintonii Sandwith, las tres primeras las pasó a sinonímia y de la cuarta él propuso e hizo una nueva combinación incluyéndola como a una variedad L. rugosus Benth. var. hintonii (Sandwith) F.J. Herm.
Sousa (1987) incluye a L. apricus Lundell dentro de $L$. rugosus Benth., pero dándole la categoría infraespecífica de subespecie, reconociendo que dentro de la variabilidad de $L$. rugosus, hay unidades discretas que se agrupan en taxa discernibles.

\section{Resultados}

En este trabajo se reconocen como subespecies de L. rugosus, además de la típica, a Lonchocarpus apricus Lundell, L. gillyi Lundell y una nueva; pero tanto L. hidalgensis Lundell como L. hintonii Sandwith se les considera especies emparentadas a $L$. rugosus, pero diferentes, para contrastar a estas dos con $L$. rugosus, se elaboró la siguiente clave.

\section{Clave de las Especies Incluidas por Hermann en Lonchocarpus rugosus}

1. Pelosidad de los brotes vegetativos largo pilosa hasta $2 \mathrm{~mm}$ de largo. Estipulas acintado-cuspidadas a laminado-obovadas, de (4-) 6-19 mm de largo, generalmente reflexas, a patentes, persistentes a caducas. Floración generalmente tardía. Estandarte adaxialmente densamente cobrizo seríceo, abaxialmente glabro, rojo a guinda.

L. rugosus Benth.

1. Pelosidad de los brotes vegetativos corto seríceo a corto tomentoso a velutino, hasta $0.6 \mathrm{~mm}$ de largo. Estípulas aciculares a linear-subuladas, de 1.5-4.5 mm de largo, rectas, pronto caducas. Floración precoz a coetánea. Estandarte adaxialmente moderadamente pardo-amarillento a canescente seríceo, adaxialmente glabro, morado, violeta o púrpura.

2. Pelosidad de los brotes vegetativos corto seríceo. Folios hasta $3.5 \mathrm{~cm}$ de ancho; estípulas linear-subuladas 3.5-4.5 mm largo. Floración precoz; flores 6-7 mm de largo; frutos coriáceos, las valvas aplanadas y lisas a la altura de las semillas; plántulas con los cotiledones epigeos.

L. hintonii Sandwith

\footnotetext{
${ }^{1}$ Departamento de Botánica, Instituto de Biología, Universidad Nacional Autónoma de México, Apdo. Postal 70-367, 04510 México, D. F.sousa@servidor.unam.mx
} 
2. Pelosidad de los brotes vegetativos corto tomentoso a velutino. Folíolos hasta $1.6 \mathrm{~cm}$ de ancho; estípulas aciculares 1.5.2.2 mm de largo. Floración coetánea; flores 8-11 mm de largo; frutos subcoriáceos, las valvas abombadas a la altura de las semillas; plántulas con los cotiledones hipogeos.

L. hidalgensis Lundell

Lonchocarpus rugosus Benth. J. Linn. Soc., Bot. 4 (Suppl.): 92. 1860. Tipo: México. Campeche: W. Houstoun s.n. (holotipo, BM!).

Árboles 6-25 (-45) m de alto, caducifolios; corteza exterior inicialmente lisa, posteriormente rugosa, corteza interior sin producir fluido resinoso al corte; ramas jóvenes densamente ferrugíneo pilosas, los pelos hasta $2 \mathrm{~mm}$ de largo, posteriormente glabrescentes, lenticelas numerosas, orbiculares a elíptico-oblongas, oscuras; estípulas acintadocuspidadas, laminar-obovadas, de (4-) 6-15 (-19) mm de largo, en la base, de 0.5-2 (-4) mm de ancho, en el ápice mucronado a subulado, patentes a reflexas, pronto caducas a persistentes; pecíolo 2.5- 4 (5.5) cm de largo; raquis foliar peloso como las ramas, 6-12 (-21) cm de largo; hojas 9-21 (27) -folioladas; folíolos (0.7-) 2.5-6 (-8.5) cm de largo, (0.4-) 1-3 (4.5) cm de ancho, elípticos a ligeramente ovados a ligeramente obovados, oblongos a angostamente oblongos en ocasiones lanceolados, la base redondeada a ligeramente cuneada y frecuentemente asimétricos, el ápice obtuso a en ocasiones agudo y frecuentemente mucronados, subcoriáceos a coriáceos, sin punctuaciones pelúcidas, opacos y moderadameante ferrugíneo a canescente velutinotomentosos, en ocasiones esparcidamente por el haz y densamente a moderadamente en el envés; nervadura primaria, secundarias y terciarias profundamente impresas en el envés; nervaduras laterales (5-) 7-11 (-15). Inflorescencias 4-14 (-18) cm de largo, simples, axilares y paniculáceas, erectas, densifloras, floración generalmente tardía; pedunculadas, pedúnculos florales 0.71.2 (-1.7) mm de largo; pedicelos 1- $2.5 \mathrm{~mm}$ de largo, bractéolas opuestas, insertadas ligeramente abajo de la base del cáliz y por debajo del ápice del pedicelo, lineares, 1- 2.1 $\mathrm{mm}$ de largo. Flores 7-9 (-10) mm de largo; cáliz ligeramente zigomorfo, epuncteado, el tubo 2-4 mm largo, 3-5 $\mathrm{mm}$ de ancho, los 2 dientes vexilares obtusos y unidos, los 3 carinales triangular-agudos y libres, el diente central generalmente más largo que los laterales; corola roja, rojo ladrillo, marrón a púrpura oscuro, epuncteada, todos los pétalos pardo-amarillento-cobrizo seríceos adaxialmente; estandarte reflexo, algo cóncavo, la lámina oblata a orbicular, 7-9 (-10) mm de ancho; anteras glabras; ovario densamente seríceo, 4-7- ovulado. Legumbre indehiscente 5-15 cm de largo, 1.2- $3 \mathrm{~cm}$ de ancho, aplanada, elíptica cuando 1-semilla, oblongo-elíptica a oblonga cuando 2 o más semillas, cartácea, pardo-amarillenta, la base atenuada a subsésil, el ápice rostrado, obtuso, atenuado, con 1-3 (-5) semillas; valvas aplanadas lisas o rugosas o abombadas a la altura de las semillas, los márgenes constrictos entre las semillas, o rectos, densa a moderadamente pardoamarillento a ferrugíneo velutino a piloso o seríceo; márgenes vexílar y carinal angostamente aquillados; semillas 1-3(-6) por fruto, 6-10 mm largo, amarillo-rojizas a castaño, en ocasiones castaño oscuro. Plántulas con cotiledones epigeos o hipogeos, eofilos opuestos, 3foliolados.

\section{Clave de las Subespecies de Lonchocarpus rugosus}

1a. Estípulas acintado-cuspidadas, frecuentemente patentes, pronto caducas, tricomas de brotes vegetativos hasta $1.2 \mathrm{~mm}$ de largo.

2a. Folíolos hasta $1.6 \mathrm{~cm}$ de ancho, legumbres hasta $1.9 \mathrm{~cm}$ de ancho, valvas abombadas a la altura de las semillas; márgenes rectos; plántulas con los cotiledones hipogeos.

L. rugosus Benth. subsp. gillyi (Lundell) M. Sousa

2b. Folíolos hasta $4 \mathrm{~cm}$ de ancho; legumbres hasta $3 \mathrm{~cm}$ de ancho, valvas rugosas a la altura de las semillas, márgenes constrictos; plántulas con los cotiledones epígeos.

L. rugosus Benth. subsp. apricus (Lundell) M. Sousa

1b. Estípulas laminado-obovadas, frecuentemente reflejas, más o menos persistentes; tricomas de brotes vegetativos hasta 2 mm de largo. 
Sousa S., M.: Las subespecies de Lonchocarpus rugosus Benth. (Leguminosae, Papilionoideae: Millettieae)

3a. Hojas 15-19-folíololadas; folíolos hasta $2 \mathrm{~cm}$ de ancho, lóbulo central carinal del cáliz, ligeramente más largo que los laterales o casi tan largo como ellos; plántulas con los cotiledones epígeos. L. rugosus Benth. subsp. stipulaceus M. Sousa

3b. Hojas 9-13(-17)-folioladas; folíolos hasta $4.5 \mathrm{~cm}$ de ancho, lóbulo central carinal del cáliz, mucho más largo que los laterales; plántulas con los cotiledones hipogeos.

L. rugosus Benth. subsp. rugosus

Lonchocarpus rugosus Benth. subsp. apricus (Lundell) M. Sousa, Fl. Novo-Galiciana 5: 573. 1987. Lonchocarpus apricus Lundell, Lloydia 2: 90. 1939 . Tipo, México, Chiapas, Finca Esperanza, Escuintla, E. Matuda S-197 (holotipo, MICH!; isotipos, A!, F!, MO!, US!, VT!). Ilustración: Fig. 55. Witsberger et al.1982. [1983] (como Lonchocarpus rugosus Benth.).

Lonchocarpus stenodon Harms, Fedde, Rep. Spec. Nov. 17: 324. 1921. Tipo: México. Oaxaca: Pinotepa, H. Galeotti 3459 (holotipo, BR!; isotipos, F! (fragmento), NY!).

Nombres comunes: Palo de aro (Edo. de México); matabuey, pellejo de vieja (Oaxaca); iit'it'ul, matabuey (Chiapas); chaperno, matabuey (Guatemala); chapulaltapa (El Salvador); cincho, coyote, quebracho (Honduras).

Árboles hasta $20 \mathrm{~m}$ de alto; tricomas de brotes vegetativos hasta $1.2 \mathrm{~mm}$ de largo. Hojas (9-) 11-17 (-19) folioladas; folíolos (0.4-) 1-2.5 (-4) cm de ancho, elípticos a oblongos; estípulas (4-) 6-10 (-15) x 1-2 (-3) mm en la base, pronto caducas; cáliz con el lóbulo carinal central mucho más largo que los laterales; ovario (4-) 5-7- ovulado. Legumbre 2-3 cm de ancho, valvas rugosas a la altura de las semillas, márgenes constrictos. Plántulas con los cotiledones epigeos.

Distribución, hábitat y fenología. De México a Honduras; en México de Jalisco, Colima, Michoacán, Edo. de México, Guerrero, Morelos, Puebla, Oaxaca y Chiapas, en Centroamérica en Guatemala, El Salvador y Honduras, se trata de una de las subespecies con mayor distribución. Habita en diversos tipos de vegetación, muy frecuente en selvas caducifolias, selvas medianas subperennifolias, en vegas de ríos, bosques de galerías y en áreas templadas con bosques de Quercus y Quercus-Pinus. En altitudes de 101400 (-1700) m. La floración, se inicia a principios de junio y termina a mediados de agosto, aunque en ocasiones concluye a finales de septiembre y principios de octubre; fructifica a principios de septiembre y concluye a finales de marzo del siguiente año.
Material representativo. MÉXICO, Jalisco, Mpio. Pihuamo: desviación a Buenpais, car. Tecalitlán-Pihuamo, J. Arturo S. Magallanes 1796 (MEXU); Mpio. Pihuamo: Road to Mina Las Encinas, at crossing of Río Barreras near the high bridge a few miles south of Pihuamo, altitud $400 \mathrm{~m}$, Rogers McVaugh 1486 (MEXU, MICH). Colima, Carretera a Minas "Las Encinas", Faustino Miranda 9073 (MEXU). Michoacán, Mpio. Aguililla: Las Cruces, $19 \mathrm{~km}$ al N de Aguililla, altitud 800 m, Mario Sousa S. 10750 (MEXU); Mpio. Aquila: Barranco del Potrero, $5 \mathrm{~km}$ arroyo arriba, altitud $210 \mathrm{~m}$, B. Guerrero C. 70 (MEXU); Mpio. Arteaga: $22 \mathrm{~km}$ al SO de Arteaga, car. Nva. Italia-Playa Azul, José Carmen Soto Núnez 1362 (MEXU); idem, Sierra Madre del Sur, altitud 800 m, Sousa 8037 (MEXU). Mpio. Chinicuila: cerca del Ocotillo, $1-5 \mathrm{~km}$ al SO de Villa Victoria, altitud 715-1200 m, Soto N. 2523, 2551 (MEXU); Mpio. Lázaro Cárdenas: car. Playa Azul-Nueva Italia a $11 \mathrm{~km}$ al $\mathrm{N}$ de Playa Azul, M. Ladd O. 289 (MEXU). Estado de México, Mpio. Tejupilco de Hidalgo: Acatitlan, Tejupilco, Temascaltepec, G. B. Hinton 5891 (A, F, MICH); Mpio. Tejupilco de Hidalgo: Barranca de Los Muñecos, S. de Nanchititla, altitud 700 m, Carmen Zepeda Gómez 470 (MEXU); Mpio. Tejupilco de Hidalgo: Plaza de Gallos, Tejupilco, Temascaltepec, Hinton 6325 (A, F, US); Mpio. Tonatico, Ojo de Agua, Tonatico, altitud 1100 m, Eizi Matuda 27958 (MEXU). Morelos, Mpio. Cuernavaca: km 20 car. Cuernavaca-Cuautla, Cañon de Lobos, Marina Jacobo Curiel s.n. (MEXU); Mpio. Cuernavaca: Puente de la Muerte, Palmira, vega del Río del Pollo, a $5 \mathrm{~km}$ al S de Cuernavaca, altitud $1200 \mathrm{~m}$, Sousa 3799, 3841, 10661 (MEXU); Mpio. Cuautla: Oaxtepec, F. Gallegos Harking s.n., 318 (MEXU); Mpio. Tlaquiltenango: SE de Quilamula rumbo a "El Potrero", altitud 1100 m, B.J. Maldonado A. 5311 (MEXU). Puebla, Mpio. Izúcar de Matamoros: Paraje Infiernillo $12 \mathrm{~km}$ al SE de Raboso, altitud $1450 \mathrm{~m}$, Enrique Guízar Nolazco 1057, 1109 (MEXU); Mpio. Izúcar de Matamoros: $21 \mathrm{~km}$ al S de Izúcar de Matamoros car. a Oaxaca, altitud 1300 m, Pedro Tenorio Lezama 14186 (MEXU); Mpio. Jolalpan: Ranchería Azoquitempa, Eleno Moreno Macías 92 (MEXU); Mpio. Jolalpan: orilla del camino Zacacuautla-El Tehuixtle, altitud 1140 m, Guízar 
1545 (MEXU). Guerrero, Mpio. Atenango del Río: 3 km al NE de Atenango del Río, altitud $661 \mathrm{~m}$, Ofelia Delgado Hernández 313 (MEXU); Mpio. Atenango del Río: 1 km al NE de Atlapa, altitud 704 m, Delgado 1022 (MEXU); Mpio. Chilpancingo: Rincón de la Vía, Hubert Kruse 3720 (MEXU); Mpio. Chilpancingo: Cañada de Azizintla al N de Chilpancingo, Javier Chavelas Polito 3201 (A, MEXU, US); Mpio. Huitzuco: $43 \mathrm{~km}$ al O-NO de Chaucingo, altitud 850 m, Sousa 13229 (MEXU); Mpio. Iguala: Cañón de la Mano Negra, entre Los Amates y El Naranjo, $10 \mathrm{~km}$ al N de Iguala por el ferrocarril, altitud 900-1000 m, C. Catalán H. 430 (MEXU); Mpio. Iguala: Iguala, altitud $3000 \mathrm{ft}, C$. G. Pringle 10338 (COLO, DUKE, F, GH, IJ, MICH, SMU, TEX, US); Mpio. Iguala: Cañon de la Mano Negra, near Iguala Canyon, J. N. Rose, 9380 (F, NY, US). Mpio. Iguala: Cañon de los Sabinos a $7 \mathrm{~km} O$ de Pachidia, al O de Iguala, altitud 1130 m, Sousa 3826 (A, MEXU, US); Mpio. José Azueta: car. a Cd. Altamirano por el camino que va a La Vainilla, altitud 70 m, Susana Peralta G. 388 (MEXU); Mpio. Olinalá: $4 \mathrm{~km}$ al $\mathrm{N}$ de Santa Gertrudis, camino Olinalá-Papalutla, ladera Sur, altitud 1300 m, José Luis Contreras Jiménez 1794 (MEXU); Mpio. Taxco de Alarcón: $14 \mathrm{~km}$ al S de Casahuatlan, altitud 1520 m, Jorge Calónico Soto 7014 (MEXU); Mpio. Tecpan: El Campamento, 2 km de El Porvenir, altitud 780 m, Soto N. 12320 (MEXU). Oaxaca, Distr. Jamiltepec, Mpio. San Sebastian Ixcapa: 9 $\mathrm{km}$ al SE de San Sebastian Ixcapa, altitud $100 \mathrm{~m}$, M. Sousa S. 8458 (MEXU); Distr. Juchitan, Mpio. El Barrio de La Soledad: a $11 \mathrm{~km}$ al S de El Barrio car. Est. Microondas, altitud 450 m, Sousa 8751 (MEXU); Distr. Juchitan, Mpio. El Barrio de La Soledad: Ajal, $11 \mathrm{~km}$ al S-SE de Matias Romero, altitud 60-150 m, Sousa 9197, 10215 (MEXU); Distr. Juchitan, Mpio. San Miguel Chimalapa: $2 \mathrm{~km}$ al S de la Congregación Benito Juárez, valle del Río Portamonedas, car. $36 \mathrm{~km}$ en línea recta al $\mathrm{N}$ de San Pedro Tapanatepec, altitud 900 m, Salomón Maya J. 309 (MEXU); Distr. Juchitan, Mpio. San Miguel Chimalapa: Río Escondido (Arroyo Baúl) $0.1-1 \mathrm{~km}$ al $\mathrm{O}$ de su unión con el Río Portamonedas y de Benito Juárez, 30 a 38 km en línea recta al N de San Pedro Tapanatepec, altitud 900 m, Maya 1968, 2187 (MEXU); Distr. Juchitan, Mpio. San Miguel Chimalapa: $2 \mathrm{~km}$ al SO de San Miguel Chimalapa, altitud 110 m, Sousa 8700 (MEXU); Distr. Juchitan, Mpio. San Pedro Tapanatepec: Humoa, a $14 \mathrm{~km}$ al NE de San Pedro Tapanatepec, altitud 500 m, Sousa 11304 (MEXU); Distr. Juchitan, Mpio. Santa Ma. Chimalapa: Arroyo Piedras Anchas, afluente del Río Portamonedas, que desemboca ca. 6 km en línea recta al $\mathrm{N}$ de San Pedro Tapanatepec, cerro al N, altitud 1000 m, Maya 2506 (MEXU); Distr. Juchitan,
Mpio. Santa Ma. Chimalapa: ca. $6 \mathrm{~km}$ en línea recta al NE de Benito Juárez, ca. 43 km en línea recta al N de San Pedro Tapanatepec, arroyo entre La Esperanza y Arroyo de Los Sastres, altitud 1000-1100 m, Maya 3798 (MEXU); Distr. Juchitan, Mpio. Santa Ma. Chimalapa: 5 km N del poblado Benito Juárez, altitud 780 m, Silvia H. Salas M. 1105 (MEXU); Distr. Juchitan, Mpio. Santo Domingo Zanatepec: $13 \mathrm{~km}$ al NO de Tapanatepec, cerca al O del General Pascual Fuentes, altitud 60 m, Sousa 3812 (A, MEXU, US); Distr. Juchitan, Mpio. Santo Domingo Zanatepec: San Pedro Las Palmas, $16 \mathrm{~km}$ al S de Santo Domingo Zanatepec, altitud 500 m, Sousa 7384 (MEXU); Distr. Juquila, Mpio. San Gabriel Mixtepec: $24 \mathrm{~km}$ al N de Puerto Escondido, altitud 300 m, Sousa 3703 (MEXU); Distr. Juquila, Mpio. San Pedro Juchatengo: km 178 de la car. México 131 aprox. $11 \mathrm{~km}$ al S de Ojo de Agua, altitud $1000 \mathrm{~m}$, A. Reyes García 2944 (MEXU); Distr. Pochutla, Mpio. San Miguel del Puerto: Sta. Ma. Xadani, 5 km al N, altitud 500 m, Salas M. 3006, 3337 (MEXU); Distr. Pochutla, Mpio. San Pedro Pochutla: Lagunilla, $8 \mathrm{~km}$ al N de Pochutla, altitud $190 \mathrm{~m}$, Sousa 5336 (MEXU); Distr. Pochutla, Mpio. San Pedro Pochutla: Toltepec, al N de Chacalapa, Rafael Torres Colín 6823 (MEXU); Distr. Pochutla, Mpio. Santa María Huatulco: Cerro Apango (Huatulco), altitud $600 \mathrm{~m}, C$. Conzatti 3115 (MEXU); Distr. Sola de Vega, Mpio. San Miguel Sola de Vega: a $6 \mathrm{~km}$ al SO, o en Ojo de Agua, entre 24 a $33 \mathrm{~km}$, al SO de Sola de Vega, al N de Puerto Escondido, altitud 1000-1250 m, Sousa 7646, 9987, 10519 (MEXU). Chiapas, Mpio. Amatenango de la Frontera: 2 km al $\mathrm{N}$ de Amatenango, altitud $930 \mathrm{~m}$, Esteban M. Martínez Salas 22114 (MEXU); Mpio. Angel Albino Corzo: 3-5 km above Jaltenango, altitud $900 \mathrm{~m}$, D. E. Breedlove 38641 (MEXU); Mpio. Angel Albino Corzo: slopes of Río Cuxtepec, below Finca Cuxtepec, altitud 900 m, Breedlove 52116 (MEXU); Mpio. Angel Albino Corzo: Independencia a Guadalupe Victoria, Cuxtepeques, J. Chavelas 4451 (MEXU); Mpio. Angel Albino Corzo (Jaltenango): Piedra Blanca a $12 \mathrm{~km}$ al N-NO de Nuevo Vicente Guerrero, altitud 550 m, Sousa 11507 (MEXU); Mpio. Arriaga: 6 km $\mathrm{N}$ of Arriaga, altitud $250 \mathrm{~m}$, Breedlove 20925 (MEXU); Mpio. Arriaga: $2.8 \mathrm{~km}$ al NE de Monte Bonito, car. Tierra y Libertad-Arriaga, altitud $420 \mathrm{~m}$, Gabriel Flores Franco 5314 (MEXU); Mpio. Arriaga: en el puente El Toronjal, sobre la car. México 190, altitud 450 m, Reyes 3955, 5322 (MEXU); Mpio. Arriaga: en Poza Galana, altitud $400 \mathrm{~m}$, Reyes 5355 (MEXU); Mpio. Arriaga: La Bondad 6 km al N de Arriaga, altitud $100 \mathrm{~m}$, Sousa 4246 (MEXU); Mpio. Arriaga: $1 \mathrm{~km}$ al $\mathrm{O}$ de Río Las Arenas y $11 \mathrm{~km}$ al O de Arriaga, altitud 10 m, Sousa 7396 (MEXU); Mpio. Arriaga: 
Sousa S., M.: Las subespecies de Lonchocarpus rugosus Benth. (Leguminosae, Papilionoideae: Millettieae)

Sta. Isabel $2 \mathrm{~km}$ al S de Tierra y Libertad, altitud $720 \mathrm{~m}$, Sousa 13119 (MEXU); Mpio. Chiapa de Corzo: en la barranca El Chorreadero, a 25 km al E de Tuxtla Gutiérrez, sobre la car. a San Cristóbal de las Casas, Edgar Cabrera C. 5931 (MEXU); Mpio. Chiapa de Corzo: above El Chorreadero, altitud 800 m, Breedlove 20437 (MEXU); Mpio. Chicoasen: Close to the mirador "Manos Que Imploran" which lies on a side road off the main road running $\mathrm{N}$ from Tuxtla Gutiérrez to Chicoasen, altitud 600 m, Colin E. Hughes 1489 (MEXU); Mpio. Cintalapa: Las Perlas, al NE de Rizo de Oro, km 33-28 de la car. México 190, altitud 803 m, Leonardo Alvarado C. 210 (MEXU); Mpio. Cintalapa: al SO de Rizo de Oro, car. México 190 entre km 28 - 29, altitud 780 m, Alvarado 514 (MEXU); Mpio. Cintalapa: 12 km S of Mexican hwy. 190 near Rizo de Oro, near the microwave station of La Mina, crest of the Sierra, altitud 1000 m, Breedlove 20673 (MEXU); Mpio. Cintalapa: on the road from Triunfo de Madero to Cal y Mayor $1 \mathrm{~km}$ to the $\mathrm{N}$ of San Sebastian and $33 \mathrm{~km}$ to the NO of Cintalapa, altitud 600 m, D. J. Macqueen 297 (MEXU); Mpio. Cintalapa: Ejido La Majada, altitud 540 m, Reyes 6883 (MEXU); Mpio. Cintalapa: Rancho La Cabaña, altitud 950 m, Reyes 6910 (MEXU); Mpio. Comitán de Domínguez: $5 \mathrm{~km}$ al $\mathrm{N}$ de J. Mújica camino a Tzimol, altitud 800 m, Martínez 22071 (MEXU); Mpio. Comitán de Domínguez: en Santa Rita (Trapichito) $2.5 \mathrm{~km}$ al NO de Uninajab, altitud $1150 \mathrm{~m}$, Reyes 1842 (MEXU); Mpio. Comitán de Domínguez: $8 \mathrm{~km}$ al SO de Tzimol, altitud 800 m, Soto N. 13436 (MEXU); Mpio. Comitán de Domínguez: SE de Trinitaria, Comitán, altitud 1310 m, Sousa 2704 (MEXU); Mpio. Escuintla: Valle de Cintalapa, cerca de Escuintla, Hernández 236 (MEXU); Mpio. Escuintla: Esperanza, Escuintla, altitud 160 m, Matuda 1788, 2620, 4020 (MEXU); Mpio. Escuintla: Piñuela, Escuintla, altitud 500 m, Matuda 18760-A (MEXU); Mpio. Frontera Comalapa: $9 \mathrm{~km}$ al NE de Amatenango de la Frontera, car. Motozintla-Comalapa, altitud $670 \mathrm{~m}$, Alfonso Delgado S. 806 (MEXU); Mpio. Frontera Comalapa: a $5 \mathrm{~km}$ al O de Frontera Comalapa, camino a Chicomucelo, altitud $600 \mathrm{~m}$, Sousa 11848 (MEXU); Mpio. Frontera Comalapa: Rancho Ley, a $4 \mathrm{~km}$ al $\mathrm{O}$ de Frontera Comalapa, en el camino Chicomucelo, altitud 850 m, Sousa 12886 (MEXU); Mpio. Jiquipilas: Quintana Roo, altitud 600 m, O. Farrera S. 215 (MEXU); Mpio. Jiquipilas: Ejido Niños Héroes, $4 \mathrm{~km}$ al S del entronque a Las Minas de la car. Juchitán-Tuxtla Gutiérrez, altitud 630 m, Flores 5285 (MEXU); Mpio. La Concordia: $96 \mathrm{~km} \mathrm{~S}$ of mexican hwy. 190 on road to Nuevo Concordia, altitud 750 m, Breedlove 38520 (MEXU); Mpio. La Trinitaria: along small dirt road to Boquerón \& Ejido
Mújica W of Mexican hwy. 190 at point $18 \mathrm{~km} \mathrm{SW}$ of La Trinitaria, altitud 900 m, Breedlove 42327, 42385 (MEXU); Mpio. La Trinitaria: 10-13 km S-SE de la Trinitaria, altitud 1000-1200 m, Grether 1380, 1728, 1735 (MEXU); Mpio. La Trinitaria: a 30-35 km al N de Cd. Cuauhtémoc sobre el camino hacia la Trinitaria, Téllez 7090 (MEXU); Mpio. La Trinitaria: 5.5 mi SE of La Trinitaria, altitud 3900 ft, Grady L. Webster 17930 (MEXU); Mpio. Ocozocoautla de Espinoza: 3 - 5 km SE of Ocozocoautla along Mexican hwy. 190, altitud $950 \mathrm{~m}$, Breedlove 23399 (MEXU); Mpio. Pijijiapan: vega Río Pijijiapan, Hernández 248 (MEXU); Mpio. Pijijiapan: Puente "Las Margaritas" $11 \mathrm{~km}$ al SE de Pijijiapan, altitud $30 \mathrm{~m}$, Sousa 4240 (MEXU); Mpio. Pijijiapan: 5 km al NO Pijijiapan, altitud 40 m, Sousa 4243 (MEXU); Mpio. San Fernando: en cañada, carretera a San Fernando, Miranda 5126 (MEXU); Mpio. Siltepec: Malpaso, Siltepec, Matuda 4525 (MEXU); Mpio. Siltepec: 6-12 km al O de Siltepec, cerca del Barrio Parralito, altitud 1170 m, Reyes 1581, 1583 (MEXU); Mpio. Socoltenango: $30 \mathrm{~km}$ E SE of Pujiltic on road to Comitán, altitud $762 \mathrm{~m}$, Breedlove 53625 (MEXU); Mpio. Socoltenango: 3 km al E de Pujiltic (Pojiltic); altitud 700 m, Sousa 6732 (MEXU); Mpio. Tapachula: Los Llanos Tapachula, near beach, Matuda 18507 (MEXU); Mpio. Tapachula: Paraste, NE de Tapachula, altitud $240 \mathrm{~m}$, Sousa 3814 (MEXU); Mpio. Tenejapa: Jeshab, altitud 700 m, Alush Méndez Ton 6498 (MEXU); Mpio. Tonala: Cerro Bernal, ca. $25 \mathrm{~km} \mathrm{SE} \mathrm{of}$ Tonala area of microwave station Quetzalapa, altitud $500 \mathrm{~m}$, Gerrit Davidse 30146 (MEXU); Mpio. Tonala: en la estación de microondas Quetzalapa, en las faldas del Cerro Bernal, a $29 \mathrm{~km}$ al SE de Tonala camino a Tapachula, altitud 400 m, Martínez 8722 (MEXU); Mpio. Tonala: en las cercanías de la zona arqueológica Iglesia Vieja, altitud 750 m, Reyes 5609 (MEXU); Mpio. Tuxtla Gutiérrez: Pishtimbak, Enrique López 7524 (MEXU); Mpio. Tuxtla Gutiérrez: en cañada Chacona-Aguacate, NO Tuxtla Gutiérrez, Miranda 6486 (MEXU); Mpio. Tuxtla Gutiérrez: El Zapotal al SE de Tuxtla Gutiérrez, altitud 660 m, E. Palacios E. 312 (MEXU); Mpio. Tuxtla Gutiérrez: cañón del Sumidero, $17 \mathrm{~km}$ al $\mathrm{N}$ de Tuxtla Gutiérrez, en el Mirador La Coyota, altitud 1220 m, Reyes 1445 (MEXU); Mpio. Tzimol: a $80 \mathrm{~km}$ al SE de Villa las Rosas, camino a Tzimol, altitud 800 m, Martínez 20219 (MEXU); Mpio. Tzimol: a 4 km al N de Tzimol, altitud 1240-1255 m, Reyes 185, 186, 191, 194 (MEXU); Mpio. Tzimol: 5 k. al SO de Tzimol, altitud 1150 m, Sousa 11317, 11894 (MEXU); Mpio. Villa de Corzo: near Colonia Vicente Guerrero, altitud 920 m, Breedlove 48602 (MEXU); Mpio. Villa de Corzo: Rancho El Retiro, altitud 769 m, Emerit Meléndez 
López 606 (MEXU); Mpio. Villa Flores: 10-12 km SO of Colonia Agrónomos Mexicanos along a zigging road to the ridges of Cerro Tres Picos, altitud $1000 \mathrm{~m}$, Breedlove 30241 (MEXU). GUATEMALA, Baja Verapaz, $15 \mathrm{~km} \mathrm{~S}$ of Granados, W. E. Harmon 4358 (ENCB, MO). Chiquimula, $12 \mathrm{~km} \mathrm{~N}$ of Esquipulas on road to Guatemala, altitud $710 \mathrm{~m}$, Hughes 278 (MEXU); betw. La Laguna and Chiquimula, along Río Taco, altitud 500-1000 m, Julian A. Steyermark 30740 (F). El Progreso, a $21 \mathrm{~km}$ al SO de El Rancho, camino a la cd. de Guatemala, altitud 1000 m, Martínez 23260 (MEXU); El Aserradero, altitud 850 m, Sousa 4223 (MEXU). Escuintla, San Antonio Jute, Paul C. Standley 64870 (A). Guatemala, Concua Bridge over Río Motagua, road betw. Guatemala and Rabinal, altitud 300 m, Standley 59303 (A, F). Huehuetenango, betw. Cuilco and aldea of San Juan, $2.5 \mathrm{mi}$. W of Cuilco along Río Cuilco, altitud 1200-1300 m, Julian A. Steyermark 50853 (F, MO); Paso del Boquerón, below La Libertad, along Río Trapichillo, below La Libertad, altitud 1200-1300 m, Steyermark 51207 (F, MO); Cienaga de Lagartero, below Miramar, altitud 300 m, Steyermark 51554 (F, MO); Santa Ana Huista, altitud 500 m, Mario Véliz 13011 (MEXU). Jutiapa, Vicinity of Jutiapa, altitud 850 m, Standley 75694 (F). Santa Ana, Huista, altitud 500 m, Véliz 13011 (MEXU). Retalhuleu, about $7 \mathrm{~km} \mathrm{~W}$ of Coyotenango, banks of the Río Samalá, altitud 330 m, Hughes 428 (MEXU); vicinity of Retahuleu, altitud $240 \mathrm{~m}$, Standley 88721 (F). San Marcos, finca Vergel near Rodeo, altitud 900 m, Standley 68895 (F). Santa Rosa, Río Esclavos, altitud $1000 \mathrm{~m}$, Heyde y Lux ex J. D. Smith 3281 (F, G, GH, US); La Joya de Limón, E of Cuilapa, altitud 900 m, Standley 78247 (A, F); at km 114 hwy. to Coban, Morazan, El Progreso, altitud 500 m, Juan J. Castillo 1558 (F). HONDURAS, Choluteca, inmediately above the village of Agua Fría, which lies in hills SE of Choluteca, about $15 \mathrm{~km} \mathrm{SE}$ of the town of Yusguare, altitud 760 m, Hughes 1074 (MEXU); El Cedrito, 8 km E San Bernardo, E. Repulski 397 (EAP, MEXU); San Marcos de Colón, $6 \mathrm{~km}$ al N de San Marcos de Colón, altitud $950 \mathrm{~m}$, Sousa 13302 (EAP, MEXU); $3 \mathrm{~km}$ O of the town of Copan close to the road towards La Florida and the Guatemalan border, altitud $600 \mathrm{~m}$, Hughes 1480 (MEXU, MO). El Paraiso, along Río Lizapa, betw. Galeras and Lizapa Grande, altitud 1000 m, Antonio Molina R. 166 (EAP, MEXU, MO, US); Mpio. Guinope, Galeras Guinope, Río Leotuna, cuenca Choluteca, altitud 820 m, Sousa 13281 (EAP, MEXU); Quebrada Dantas, $5 \mathrm{~km} \mathrm{~S}$ of Ojo de Agua, L. O. Williams y Molina R. 11226 (MO). Francisco Morazán, El Zamorano, on road to Guinope, on bank of Río Yeguare, M. Chorley 8 (MEXU); aldea Yaguasire, 10 km al S de Tegucigalpa, Ibis Colindres 2 (MEXU, MO); Mpio. Tegucigalpa: márgenes del Río Las Canoas, car. 5 km al oriente de Tegucigalpa, cuenca Río Choluteca, vertiente del Pacífico, altitud 1020 m, José L. Linares 4066 (EAP, MEXU); Mpio. Tegucigalpa: $54 \mathrm{~km}$ al $\mathrm{N}$ de TegucigalpaSan Pedro Sula, Linares 4377 (EAP, MEXU); Mpio. Cantarranas: Quebrada de Cantarranas conocida también como Río Chiquito, a $500 \mathrm{~m}$ de la entrada de Cantarranas por la car. a Talanga, altitud $710 \mathrm{~m}$, Linares 5214 (EAP, MEXU); on the road S from El Zamorano, $1 \mathrm{~km}$ to the $\mathrm{S}$ of Maraita on the road to Yauyupe, altitud $700 \mathrm{~m}$, Macqueen 130 (MEXU); in the banks of Río Jalán, E of Guaimaca, Molina R. 2926 (GH, US); valle del Zamorano, 36 km E de Tegucigalpa, altitud 1000 m, Ruben Rivera Pineda s.n. (MO). Intibuca, car. a baños públicos, $0.5 \mathrm{~km}$ de La Esperanza, altitud $1700 \mathrm{~m}$, Geovanny Rodríguez M. 76 (MEXU, MO). La Paz, $10 \mathrm{~km} \mathrm{~W}$ of La Paz on road towards Marcala, altitud 700 m, Hughes 221 (MEXU). Lempira, ca. Río Mejocote, $9 \mathrm{~km}$ de Gracias, altitud $1000 \mathrm{~m}$, Cirilo Nelson 203 (MEXU, MO); Mpio. Gracias: a 5 km N de Gracias, orilla del Río Arcagual, vertiente Atlántica, altitud $630 \mathrm{~m}$, Sousa 13333 (EAP, MEXU). Valle, Isla Tigre, near Amapala, P.C. Standley 20717 (US). EL SALVADOR, Ahuachapan, San Benito, La Poza de la Vuelta, altitud 450 m, Eliberto Sandoval 1685 (B, MEXU). Chalatenango, 5 $\mathrm{km}$ to the $\mathrm{N}$ of Tejutla on the road to El Poy and Las Palmas, altitud $480 \mathrm{~m}$, Macqueen 506 (MEXU). La Libertad, Mpio. Santa Tecla: $600 \mathrm{~m}$ al S de Santa Tecla, camino a Comasagua, altitud 1110 m, Martínez 30989 (MEXU). La Paz, Zacatecoluca, S. Calderón 309 (GH, US). San Salvador, desvío a Tonacatepeque, Cantón Las Delicias, Jorge González 163 (MEXU); Highway CAI, ca. $30 \mathrm{~km}$ E of Santa Ana on route from San Salvador, ca. $5 \mathrm{~km}$ past the turn to the archaeological site of San Andres heading towards Santa Ana, altitud 450 m, G. P. Lewis 1731 (MEXU); Ciudad Barrios, La Florida near Cd. Barrios, altitud 810 m, Alex K. Monro 3420 (B, BM). Finca Leticia, Los Planes de Renderos, altitud 980 m, Monro 3435 (B, BM); Univ. de El Salvador, creciendo dentro de la Universidad, cultivada, altitud $600 \mathrm{~m}$, Nadia Navarrete 2021 (B); near San Salvador, Standley 19254 (GH, US). Santa Ana, Candelária de la Frontera, ca. 6 km al NO de la Candelária de la Frontera, de entre la cima del Cerro El Yupe y el Cerro Monteverde, altitud 1350 m, Linares 2191 (EAP, MEXU); Mpio. Santa Ana, San Luis La Planta, alrededores de la antigua casa de máquinas, altitud $500 \mathrm{~m}$, Linares 5121 (EAP, MEXU). Sonsonate, ca. $2 \mathrm{~km}$ al SE de la Hacienda San Marcelino, campos de lava de las faldas del cerro Chino, altitud 1400 m, Linares 1502 (EAP, MEXU). 
Discusión. Esta subespecie se distingue de las demás por las legumbres con valvas rugosas a la altura de las semillas; frutos anchos y plántulas con los cotiledones epigeos.

Lonchocarpus rugosus Benth. subsp. gillyi (Lundell) $\mathrm{M}$. Sousa, comb. nov. Lonchocarpus gillyi Lundell, Wrightia 1: 56. 1945. Tipo: México, Chiapas: $2 \mathrm{~km}$ from Salto de Agua on Yajalón, C.L. Gilly y E. Hernández X. 430 (holotipo, LL!; isotipo, MICH! ). Figura 1.

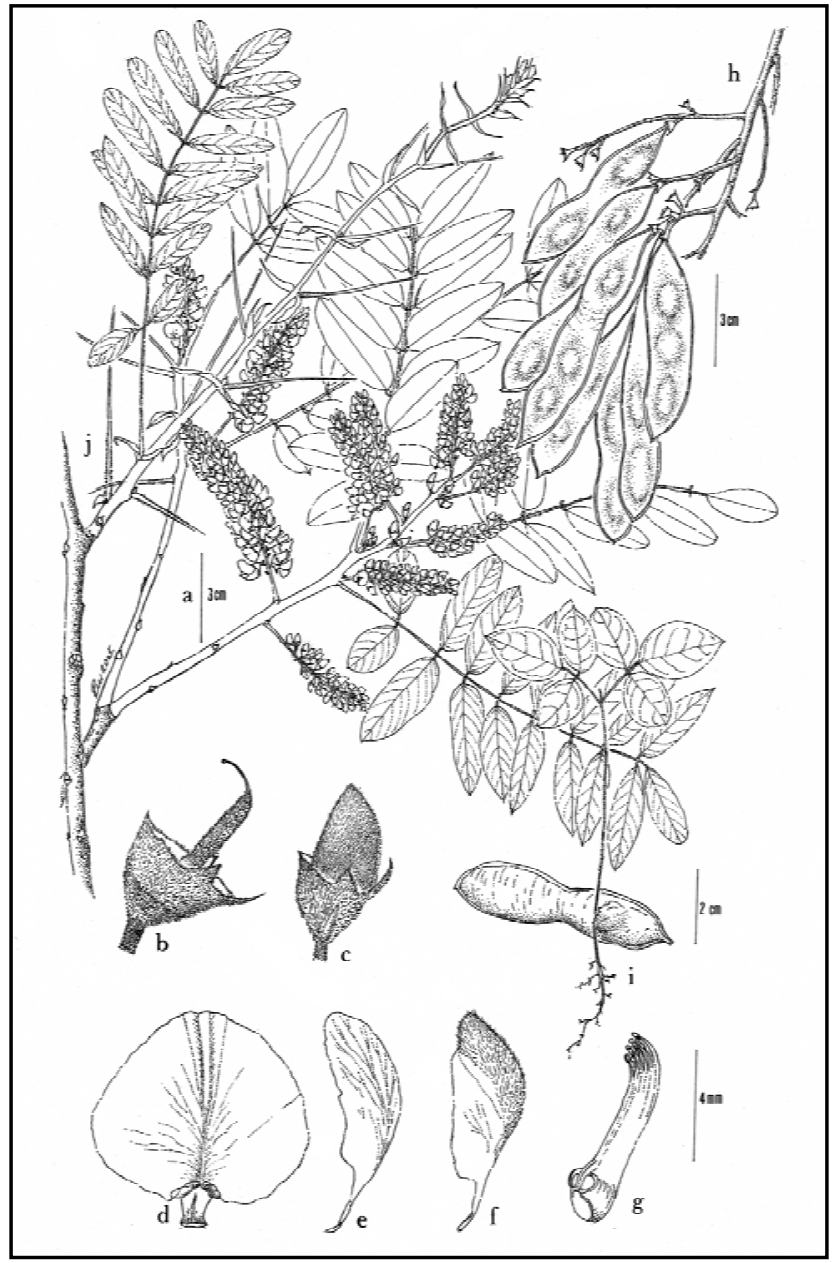

Figura 1. Lonchocarpus rugosus Benth. subsp. gillyi (Lundell) M. Sousa. A) Rama con hojas e inflorescencias. Tomado de Araque et al. 868. B) Cáliz, mostrando los lóbulos y gineceo. C) Botón floral. D) Estandarte. E) Quilla. F) Ala. G) Tubo estaminal. Tomado de D. Stevens 21642. H) Infrutescencia, mostrando los frutos. Tomado de Martínez S. 12748. I) Plántulas, mostrando cotiledones epigeos. Tomado de Sousa et al. 13355.
Nombres comunes: Gusano, biche rayo (Chiapas); macaichi (Tabasco); lán-sin (Guatemala); masicarán (Honduras).

Árboles hasta $45 \mathrm{~m}$ de alto; tricomas de brotes vegetativos hasta $1.2 \mathrm{~mm}$ de largo. Hojas 15-27-folioladas; folíolos (0.7-) 0.9-1.3 (-1.6) cm de ancho, oblongos en ocasiones elípticos; estípulas 6-11 x (0.5-) 1.2-1.5 mm (sólo en la base), pronto caducas; cáliz con el lóbulo carinal central mucho más largo que los laterales; ovario (4-) 5-6 (7)-ovulado. Legumbre 1.2-1.6 (-1.9) cm de ancho, valvas abombadas a la altura de las semillas, márgenes generalmente rectos. Plántulas con los cotiledones hipogeos.

Distribución, hábitat y fenología. Subespecie de México a Colombia; en México en Veracruz, Tabasco y Chiapas, en Centroamérica, Guatemala, Honduras, Nicaragua y Costa Rica, en Sudamérica, en Colombia. Habita en diversos tipos de vegetación de selvas altas perennifolias a bajas perennifolias, riparios, pinares tropicales, sabanoides, bosques mesófilos y encinares tropicales y templados. En altitudes de 30-900 (-1300) m. Florece de mediados de junio a finales de julio, fructifica de principios de enero a principios de noviembre.

Material representativo: MÉXICO, Veracruz, Mpio. Hidalgotitlan: 5 km NO del campamento Hermanos Cedillo por el Río Soloxuchil, altitud 150 m, M. Vázquez 1773 (MEXU). Oaxaca, Distr. Juchitan, Mpio. San Miguel Chimalapa: $2 \mathrm{~km}$ al $\mathrm{S}$ de Benito Juárez, Valle del Río Portamonedas, altitud 900 m, S. Maya J. 309 (ENCB). Tabasco, Mpio. Teapa: Puyacatengo, F. Ventura A. 20599 (MEXU); Mpio. Teapa: a orillas del Río Puyacatengo, $S$. Zamudio 979 (MEXU). Mpio. Tenosique: km 28.5 car. Tenosique-Zapata, González Leija 364 (MEXU). Chiapas, Mpio. La Libertad: Ejido Morelos, altitud $60 \mathrm{~m}$, M. Sousa 3657 (MEXU); Mpio. Ocosingo: Crucero Bethel, altitud 364 m, G. Aguilar M. 3923 (MEXU); Mpio. Ocosingo: 6.3 $\mathrm{km}$ al NE del crucero de San Javier, altitud $382 \mathrm{~m}$, Aguilar 4466 (MEXU); Mpio. Ocosingo: $1.85 \mathrm{~km}$ al SE de San Javier, altitud 493 m, Aguilar 7964 (MEXU); 3 km al S del entronque a Chancala, sobre la car. Palenque-Ocosingo, Edgar Cabrera C. 5623 (MEXU); Mpio. Ocosingo: a 6.8 $\mathrm{km}$ al NO de Crucero San Javier, Sierra de la Cojolita, altitud 414 m, Jorge Calónico Soto 24173 (MEXU); Mpio. Ocosingo: $3.5 \mathrm{~km}$ al E de Lacanja Chansayab, altitud $431 \mathrm{~m}$, Calónico 24786 (MEXU); Mpio. Ocosingo: Comunidad Lacandona Naha, $27 \mathrm{~km}$ SE de Palenque, car. fronteriza hasta el crucero Chancala, después $55.6 \mathrm{~km}$ por el camino de terracería hacia Monte Libano, altitud 950 m, Alejandro 
Durán F. 425 (MEXU); $21 \mathrm{~km}$ al S de Palenque, camino a Ocosingo, altitud $390 \mathrm{~m}, \mathrm{R}$. Grether 1720 (ENCB, MEXU, XAL); Mpio. Ocosingo: $4 \mathrm{~km}$ al SE de Nvo. Guerrero, camino a Boca Lacantum, altitud $560 \mathrm{~m}$, Esteban $M$. Martínez Salas 16788 (MEXU); Mpio. Ocosingo: En crucero el Piñal camino a Chancala, Santo Domingo, altitud 450 m, Martínez 17480 (MEXU); Mpio. Ocosingo: Ojo de Agua San Javier, altitud 406 m, Martínez 35759 (MEXU); Mpio. Palenque: $2 \mathrm{~km}$ al SE de Chancala Zapote, altitud 304 m, Martínez 36467 (MEXU); Mpio. Palenque: En ejido Agua Blanca, $15 \mathrm{~km}$ al $\mathrm{O}$ de Chocoljaito, altitud $400 \mathrm{~m}$, Martínez 5966 (MEXU); Mpio. Palenque: $6 \mathrm{~km}$ al N de Soyaló, sobre la car. Bochil, altitud $1300 \mathrm{~m}$, J. Rzedowski 33632 (ENCB); Mpio. Palenque: 4 km al SE de Palenque en el Balneario Nututum, Oswaldo Téllez Valdés 847 (MEXU). GUATEMALA, Alta Verapaz: Finca Camila, altitud 800 m, I. Kunkel s.n. (USCG); betw. Campur and Socoyó, altitud $1100 \mathrm{~m}$, P.C. Standley 91720 (F). HONDURAS, Comayagua: Las Limas, J. B. Edwards 361 (A, US); 5 km $\mathrm{N}$ of Agua Dulce, D. Hazlett 1086 (MO); Edge of Lake Yajoa, R. A. Howard 606 (A); Agua Caliente, vaguada de ríos Chamo y Humuya, $35 \mathrm{~km}$ E de Lago Yojoa, altitud 220 m, Cirilo Nelson 6280 (MEXU, MO); $30 \mathrm{~km}$ al N de Ciudad Comayagua, altitud 350 m, Nelson 6866 (MEXU, MO); La Ceiba, orilla del Río Yure, altitud 200 m, Nelson 7429 (MEXU, MO); betw. Chaparral and Pueblo de Meámbar, G. Niederlem s.n. (US). Olancho: Mpio. Yocón, Platanares, car. a Mangulile, altitud 850 m, Jorge Araque 868 (MEXU); A $4 \mathrm{~km} \mathrm{Al} \mathrm{SO} \mathrm{de} \mathrm{El} \mathrm{Pataste,} \mathrm{sobre} \mathrm{el} \mathrm{camino} \mathrm{Culmi-}$ Catacamas, altitud 500 m, Martínez 12748 (MEXU, MO); $20 \mathrm{~km} \mathrm{~S}$ de Campamento, altitud 1097 m, Carlos Soto 126 (MEXU, MO); Mpio. El Rosario Chorreron, $9 \mathrm{~km}$ al SE, altitud 905 m, Mario Sousa Sánchez 13349 (MEXU); Mpio. Juticalpa, $8 \mathrm{~km}$ al $\mathrm{N}$ de La Lima, altitud $170 \mathrm{~m}$, Sousa 13346 (MEXU); Salama, en Talagua, altitud 1000 m, Sousa 13346a (MEXU); Mpio. Jano, 24 km al NE de La Unión, altitud 820 m, Sousa 13355 (MEXU). Sta. Bárbara: Lago Yojoa, Punta Gorda, altitud 650 m, J. M. MacDougal 3123 (MEXU, MO). Yoro: Close to the road running from Sabana Larga to Macora in a tributary valley running SE from the main Aguan Valley on the banks of the Río Macora, about $10 \mathrm{~km} \mathrm{NW}$ of Jocon, altitud $380 \mathrm{~m}, J$. J. Hellin 13 (MEXU); Victoria, orilla del Río Sulaco, altitud 339 m, Nelson 7179 (MEXU, MO); NICARAGUA, Chontales: $5.2 \mathrm{~km}$ NW of Quebrada Niscala, betw. Acoyapa and Morrito, altitud $75 \mathrm{~m}$, Warren Douglas Stevens 21642 (ENCB, MEXU, MO). Rivas: Isla Ometepe, volcán Concepción, altitud 100-800 m, Walter Robleto 1062 (MEXU). COSTA RICA, Alajuela: vicinity of Los
Chiles, Río Frio, R. W. Holm 726 (A); Refugio Caño Nefro, Los Chiles, San Carlos, altitud 30 m, Nelson Zamora 1374 (MO, NY). Guanacaste: near Liberia, road to Santa María, C. W. Dodge 6730 (MO); Cordillera de Guanacaste, Estación de San Ramón, Sector Góngora, altitud 600 m, Roberto Espinoza 1306 (MEXU); Sta. Rosa National Park, at E end of the park, Daniel H. Janzen 10134 (MO), 11736, 11741 (MEXU, MO); 6.9-12 miles $\mathrm{N}$ of Liberia, grows in wet part, Janzen 341, 10135 (MO); Sta. Rosa National Park, altitud 250-300 m, Ronald Liesner 5279 (MEXU, MO); 5 $\mathrm{km}$ antes de La Irma rumbo al N, L. J. Poveda 1117 (MO); Cantón de Abangares, Valle de Tempisque- Barrio Jesus, Las Juntas de Abangares, Río Sardinal, Car. Interamericana, mojones 137-138, altitud 40 m, Victor Ramírez 382 (MEXU, NY). Puntarenas: Hacienda Sta. Marta, Cascajal, vicinity of Cascajal (25 km E-SE of Puntarenas), altitud 30100 m, Richard W. Holm 245 (NY); faja costeña de Puntarenas, San Marcos, Car. Interamericana norte, $3 \mathrm{~km}$ antes del Río Guacimal, altitud 100 m, N. Zamora 2279 (NY). San José: Near Esparta, roadside, D. Spellman 522 (MO). COLOMBIA, José Celestino Mutis 4305, 4700 (US).

Discusión. Esta subespecie se caracteriza por sus folíolos numerosos y angostos; legumbres angostas y con los márgenes generalmente rectos y las plántulas con los cotiledones hipogeos. Además se trata de uno de los árboles más altos (45 m) colectados para el género Lonchocarpus.

Lonchocarpus rugosus Benth. subsp. rugosus. Ilustración: Pittier, 1917. tabla 1, fig. A. [sólo fruto].

Nombres comunes: Canasin, choyche, chu'ul panasin, kansin, (Campeche, Quintana Roo, Yucatán, Guatemala); cabbage bark, kanasin, pine-ridge (Belice).

Árboles hasta $18 \mathrm{~m}$ de alto; tricomas de brotes vegetativos hasta $2 \mathrm{~mm}$ de largo. Hojas 9-13 (-17)folioladas; folíolos 1.5-3 (-4.5) cm de ancho, elípticos en ocasiones oblongos; estípulas 8-12 x 0.8-1.4 mm en la base, laminado-obovadas, persistentes; cáliz con el lóbulo carinal central mucho más largo que los laterales; ovario 4-5ovulado. Legumbre 1.7-2.1 cm de ancho, valvas lisas, márgenes algo constrictos. Plántulas con los cotiledones hipogeos.

Distribución, hábitat y fenología. La subespecie típica se distribuye de México a Guatemala; en México en Veracruz, Tabasco, Chiapas, Yucatán, Campeche, Quintana Roo, en Centroamérica, Belice y Guatemala; subespecie casi endémica a la provincia fisiográfica de la Península de 
Sousa S., M.: Las subespecies de Lonchocarpus rugosus Benth. (Leguminosae, Papilionoideae: Millettieae)

Yucatán. Habita en selvas bajas caducifolias a medianas subcaducifolias; en suelos calizos. En altitudes de 0-450 (1100) $\mathrm{m}$. Florece de mediados de agosto a principios de enero y fructifica de mediados de noviembre a mediados de abril.

Material representativo: MÉXICO, Veracruz, Mpio. Hidalgotitlán: Hnos. Cedillo, La Escuadra por el Río Solosuchil, Tenosique-Emiliano Zapata, altitud $150 \mathrm{~m}$, Brigada Vázquez 1224 (MEXU). Tabasco, Mpio. Balancan: $2 \mathrm{~km} \mathrm{~S}$ de la N-25 sobre la W-0 rumbo a la N-20 cerca de un drene (localidad 6), Balancan, altitud $30 \mathrm{~m}, F$. Menéndez 293 (MEXU, XAL); Mpio. Balancan: $100 \mathrm{~m}$ al N de la 15$\mathrm{N}$ y a $1.5-2 \mathrm{~km}$ al O de la W-O, Balancan, A. Novelo 106, 206 (MEXU); Mpio. Centro: Brecha Nueva Vista de Tamulte de las Sabanas a $3 \mathrm{~km}$ de Tamulte, Alma D. L. Orozco-Segovia 43 (MEXU); Mpio. Emiliano Zapata: 6 km al SE de Emiliano Zapata, sobre la car. a Palenque. por La Libertad, Edgar Cabrera C. 14274 (MEXU); Mpio. Emiliano Zapata: cerca de la desv. a San Elpidio, Ma. A. Guadarrama 384 (MEXU); Mpio. Tenosique: km 1.1 de la desviación hacia Balancan, Clark P. Cowan 3302 (MEXU); Mpio. Tenosique: $26 \mathrm{~km}$ al O de Tenosique, Oswaldo Téllez Valdés 900 (MEXU); Mpio. Tenosique: $22 \mathrm{~km}$ al $\mathrm{N}$ de Tenosique, Téllez 919 (MEXU). Chiapas, Mpio. Catazaja: $18 \mathrm{~km} \mathrm{E}$ of Catazaja, altitud $60 \mathrm{~m}, D$. E. Breedlove 47231 (MEXU); Mpio. Ocosingo: Mensura de Nvo. Guerrero con Nvo. Tumbala, G. Aguilar M. 163 (MEXU); Mpio. Ocosingo: $1 \mathrm{~km}$ de Nvo. Guerrero rumbo a Palenque, altitud 225 m, Aguilar 3093 (MEXU); Mpio. Ocosingo: Ejido de Cintalapa, altitud 300 m, A. Álvarez A. 475 (MEXU); Mpio. Ocosingo: $3 \mathrm{~km}$ al E de Nvo. Fco. León camino a Nvo. Jerusalen, altitud $194 \mathrm{~m}$, Álvarez 1100 (MEXU); Mpio. Ocosingo: $4.11 \mathrm{~km}$ al O de Nva. Jerusalen, altitud $177 \mathrm{~m}$, Jorge Calónico Soto 24352 (MEXU); Mpio. Ocosingo: borde de Río Lacantum, Estación de Biología de Cha, altitud 400 m, Guillermo Ibarra M. 4021 (MEXU); Mpio. Ocosingo: la comunidad lacandona de Lacanha-Chansayab, a $130 \mathrm{~km}$ al SE de Palenque, por la car. fronteriza hasta el Crucero San Javier, después $8 \mathrm{~km}$ hacia el O, altitud $400 \mathrm{~m}$, Samuel Levy T. 255 (MEXU); Mpio. Ocosingo: 7 km al SE del Crucero de Lacanja- Chanzayab rumbo a las ruinas de Bonampak, comunidad lacandona, altitud $410 \mathrm{~m}$, J. Luis López-García 63 (MEXU); Mpio. Ocosingo: $8 \mathrm{~km}$ al NO del ejido Nvo. Fco. León, altitud 280 m, A. Luna-Gómez 5 (MEXU); Mpio. Ocosingo: $2 \mathrm{~km}$ al E de Crucero Corozal, camino Palenque-Boca Lacantum, altitud $180 \mathrm{~m}$, Esteban M. Martínez Salas 10161, 11491-A, 14382, 15340, 15357 (MEXU); Mpio. Ocosingo: en Campamento COFOLASA a $24 \mathrm{~km}$ al SE de Crucero Corozal, camino Cola Lacantum, altitud 220 m, Martínez 7720, 10377 (MEXU); Mpio. Ocosingo: $1 \mathrm{~km}$ al $\mathrm{S}$ de Naja camino a Monte Libano, altitud 990 m, Martínez 17435 (MEXU); Mpio. Ocosingo: 3 $\mathrm{km}$ al SE de Sto. Domingo camino a Lacanja Tzeltal, altitud 450 m, Martínez 18182 (MEXU); Mpio. Ocosingo: en Ixcán sobre el Río Lacantum, altitud 150 m, Martínez 25207 (MEXU); Mpio. Ocosingo: Estación Chajul, sobre el Río Lacantum, altitud 150 m, Martínez 25287, 25495, 26528 (MEXU); Mpio. Ocosingo: En Boca Lacantum sobre el Río Lacantum, altitud 120 m, Martínez 9667, 25905, 25907 (MEXU); Mpio. Ocosingo: en las orillas del lado $\mathrm{N}$ de la colonia Benito Juárez Miramar, sobre el camino a la Laguna Miramar y a Nueva Galilea, altitud 250 m, A. Reyes García 2436 (MEXU); Mpio. Ocosingo: al $\mathrm{N}$ de la Omega, Monumento Natural Yaxchilán, altitud $150 \mathrm{~m}$, Armando Rincón Gutiérrez 1072 (MEXU); Mpio. Palenque: 12 km N of Palenque along road to Catazaja, altitud $100 \mathrm{~m}$, Breedlove 49528 (MEXU); Mpio. Palenque: 35 km al SE de Palenque, camino a Chancala, Martínez 13365 (MEXU); Mpio. Salto de Agua: W of Catazaja on road to Villahermosa, altitud $100 \mathrm{~m}$, Breedlove 55308 (MEXU). Yucatán, Mpio. Celestún: 10-15 km N of Chenchucmil, Steven P. Darwin 2408, 2458 (MEXU); Mpio. Celestún: 20 $\mathrm{km}$ al $\mathrm{N}$ de Chenchucmil hacia Celestún, altitud $3 \mathrm{~m}, L$. E. Estrada 377 (MEXU); Mpio. Chochola: a la orilla del Cenote Chen Ha, altitud 14 m, Marco Antonio Pérez Lara 585 (MEXU); Mpio. Izamal: G. F. Gaumer 996 (A, DS, F, MO, US); Mpio. Maxcanú: Chenchucmil, altitud $12 \mathrm{~m}$, E. Ucan 4907 (MEXU); Mpio. Mérida: alrededores del Jardín Botánico regional del CICY, región NO de la Ciudad de Mérida, cerca de Cordemex, altitud 8-10 m, R. Duno de Stefano 1751 (CICY, MEXU); Mpio. Mérida: Jardín Botánico regional CICY, arboretum, S. Escalante 290 (MEXU); Mpio. Muna: 18 km al E car. Muna-Maxcanú, Opichen, M. Méndez 491 (MEXU); Mpio. Oxkutzcab: car. Neh-Cacab km 10, F. May 147 (XAL); Mpio. Peto: 7 km al E de Peto, altitud 35 m, Feliciano Ku y Yam 707 (MEXU); Mpio. Peto: a 2 km al N de Peto, Pérez 206, 414 (MEXU); Mpio. Santa Elena: en los alrededores de la zona arqueológica de Sayil por la car. Uxmal-Oxkutzkab, Cabrera C. 10348 (MEXU); Mpio. Santa Elena: Uxmal, Velva E. Rudd 2026 (IJ, MEXU); Mpio. Santa Elena: a 1 km de la car. Blanca de Sotuta hacia Tixcacatukub, altitud $24 \mathrm{~m}$, E. Ucán Ek 578 (XAL); Mpio. Santa Elena: Zona arqueológica de Sayil, Ucán 2192 (XAL); Mpio. Santa Elena: at entrance to Labna, $13 \mathrm{~km}$ E of Mex. 261 on new road to Labna and Sayil, John Utley 6405 (MEXU); Mpio. Tekax: $6.1 \mathrm{~km}$ al S-SE de Ayim, camino a Sudzal Chico, altitud 114 m, Demetrio Álvarez 9295 (MEXU); Mpio. 
Tekax: car. Nojalal-Sudzal-Chico, F. May 792 (MEXU); Mpio. Tekom: al SO de Valladolid, O. G. Enríquez 110, 215 (MEXU); Mpio. Ticul: Ticul, E.C. Stewart 309 (GH); Mpio. Ticum: Ruinas de Chichén Itzá, altitud $18 \mathrm{~m}, J$. S. Flores 9611 (XAL); Mpio. Tixcacalcupul: a 12 km de Tixcacalcupul rumbo a Peto, camino rancho Tutulxiú, Yaxcabá, P. Sima 428 (MEXU); Mpio. Tizimin: en la comunidad de Dzonot ake, J. A. Aguilar Zepeda 140 (MEXU); Mpio. Tizimin: $5 \mathrm{~km}$ al S de Tizimin, ca. Calakmul, Durán 1545 (MEXU); Mpio. Tunkás: en la car. Blanca Tunkás, altitud 23 m, Pedro Yam P. 51 (XAL); ídem, Ucán 1835 (XAL); Mpio. Tzucacab: 4.5-6 km al O de Tzucacab, sitio Ab(e) 166, Pérez 423, 785 (MEXU). Campeche, Mpio. Calakmul: a $8 \mathrm{~km}$ al S de Ejido Ley de Fomento Agropecuario, camino a Dos Naciones, altitud 294 m, Álvarez 3388 (MEXU); Mpio. Calakmul: 5.62 km al O del poblado Eugenio Echeverria Castellot Num. 1 (Carrizal), altitud 295 m, Álvarez 7967 (MEXU); Mpio. Calakmul: $15 \mathrm{~km}$ al N de Zoh-Laguna, Pascual Álvaro $M$. 639 (MEXU); Mpio. Calakmul: $20 \mathrm{~km}$ al S de la antigua caseta de vigilancia de la Reserva de la Biosfera de Calakmul, altitud 204 m, Erika M. Lira C. 473 (MEXU); Mpio. Calakmul: Tuxpeña, C. L. Lundell 857, 1319 (A, DS, F, GH, MEXU, MICH, MO, US); Mpio. Calakmul: km 3 al $\mathrm{S}$ de Xcan-Ha camino a Xpujil, altitud $120 \mathrm{~m}$, Estela Madrid N. 341 (MEXU); Mpio. Calakmul: a 3.4 km al SE de La Nueva Vida, altitud 250 m, Martínez 30230 (MEXU); Mpio. Calakmul: 3-9 km al NE de Narciso Mendoza, camino a El Manantial, altitud $271 \mathrm{~m}$, Martínez 30311, 30528 (MEXU); Mpio. Calakmul: car. Chetumal a Escárcega entre Xpujil aprox. $2 \mathrm{~km}$ al $\mathrm{E}$ de Conhuas, Champoton, Sima 1486 (MEXU); Mpio. Calkini: 8 km al O de Tankuche, sobre el camino Calkini-Punta Arenas, Cabrera C. 14343 (MEXU); Mpio. Calkini: camino de Tankuche a El Remate, altitud 0 m, I. Espejel 317 (MEXU, XAL); Mpio. Calkini: a $11 \mathrm{~km}$ al O de Tankuche, car. a Isla Arena, F. Tun 164 (MEXU); Mpio. Campeche: $2 \mathrm{~km}$ al E del poblado de Tikinmul, Méndez 529 (MEXU); Mpio. Campeche: Cambeon $25 \mathrm{~km}$ E de Campeche, altitud $20 \mathrm{~m}$, Sousa 3792 (A, MEXU); Mpio. Campeche: km 2 de Zodzil hacia Pomuch, altitud $20 \mathrm{~m}$, Ucan 3679 (MEXU); Mpio. Campeche: Estación de la Vida Silvestre de Hampolol, $P$. Zamora C. 4825, 4926 (MEXU); Mpio. Champoton: within the first 6 miles along road to Pixoyal, altitud $50 \mathrm{~m}$, Melissa Luckow 3010 (MEXU); Mpio. Champoton: en terraceria a Xbacab, $5 \mathrm{~km}$ de la car. Campeche- Champoton, a $14 \mathrm{~km}$ al $\mathrm{N}$ de Cantemó, Téllez 6334 (MEXU); Mpio. Cd. del Carmen: $6 \mathrm{~km}$ al E de Fco. Escárcega, sobre la car. 186 en el tramo Escárcega-Chetumal, Cabrera C. 12512 (MEXU);
Mpio. Cd. del Carmen: desviación car. Escárcega a Villahermosa rumbo a Pital, altitud 5 m, C. Chan 6089 (MEXU); Mpio. Escárcega: Campo Experimental Forestal Tropical "El Tormento" km 5 car. Escárcega a Candelaria, Javier Chavelas Polito 47, 94, 1140, 1303 (MEXU, MICH); Mpio. Escárcega: $1 \mathrm{~km} \mathrm{~N} \mathrm{Fco.} \mathrm{Escárcega,} \mathrm{Clarence} \mathrm{Dan}$ Johnson 1611-80 (MEXU); Mpio. Escárcega: Campo Experimental Forestal Tropical "El Tormento" km 5 car. Escárcega a Candelaria, Efraim Hernández X. 211 (MEXU, MICH); Mpio. Hecelchakán: 1 km al S de Hecelchakán, sobre la car. Mérida-Campeche, Rogel Villanueva 492 (MEXU); Mpio. Hopelchén: $3 \mathrm{~km}$ al $\mathrm{S}$ de Bolonchén de Rejón, Cabrera C. 10825 (MEXU); Mpio. Hopelchén: 20 $\mathrm{km}$ al S de X-Maben, camino a Xpujil, altitud $120 \mathrm{~m}$, Martínez 28634 (MEXU); Mpio. Hopelchén: Bolonchen de Rejón, orilla S del pueblo, altitud 120 m, Sousa 12428 (MEXU); Mpio. Palizada: km 198 car. a Villahermosa, altitud 6 m, Flores 10306 (MEXU); Mpio. Ulmal: 2 km de Ulmal, M. Burgos Ch. 15 (XAL). Quintana Roo, Mpio. Benito Juárez: brecha camino del Norte $13 \mathrm{~km}$ al E de Leona Vicario, Cabrera C. 457 (MEXU); Mpio. Benito Juárez: a $5 \mathrm{~km}$ al $\mathrm{S}$ de Akumal, car. Cancún-Tulum, Cabrera C. 833, 1178 (MEXU); Mpio. Benito Juárez: a 2 $\mathrm{km}$ al S de Puerto Morelos, Cabrera C. 1506 (MEXU); Mpio. Benito Juárez: Jardín Botánico CIQRO, Puerto Morelos, altitud 3 m, Escalante 200 (MEXU); Mpio. Benito Juárez: a few $\mathrm{km} \mathrm{N}$ of Puerto Morelos close to the main road towards Cancun, altitud $5 \mathrm{~m}$, Colin E. Hughes 490 (MEXU); Mpio. Benito Juárez: $2.5 \mathrm{~km}$ al $\mathrm{O}$ de entronque hacia Vallarta, partiendo de car. Cancun-Tulum, I. Omsted 339 (CICY, MEXU); Mpio. Benito Juárez: $8 \mathrm{~km}$ al S de Puerto Morelos, en la car. a Playa del Carmen, Sousa 10905 (MEXU); Mpio. Benito Juárez: zona arqueológica de Coba, Téllez 1392, 3789 (MEXU); Mpio. Cozumel: Isla de Cozumel, en los alrededores de la zona arqueológica de San Gervacio, Cabrera C. 9895 (MEXU); Mpio. Cozumel: Isla de Cozumel, $8 \mathrm{~km}$ al E de San Miguel de Cozumel, por la car. Transversal, Cabrera C. 11168 (MEXU); Mpio. Felipe Carrillo Puerto: X-Hazil Xur, $3.5 \mathrm{~km}$ hacia San Andrés, altitud 15-16 m, F. Balam 442 (MEXU); Mpio. Felipe Carrillo Puerto: $10 \mathrm{~km}$ al S de F. Carrillo Puerto en la desviación a Laguna Ocum, Cabrera C. 283 (MEXU); Mpio. Felipe Carrillo Puerto: 4-9 km al O de F. Carrillo Puerto, rumbo a Vigía Chico, Cabrera C. 419, 1106 (MEXU); Mpio. Felipe Carrillo Puerto: a $1 \mathrm{~km}$ al S de las ruinas de Chunyaxche, Cabrera C. 953 (MEXU); Mpio. Felipe Carrillo Puerto: 8-11 km al SE de la desviación a Chumpon sobre el nuevo camino a Vigía Chico, Cabrera $C$. 3548, 4030 (MEXU); Mpio. Felipe Carrillo Puerto: en la 
zona arqueológica de Muxil (Chunyaxche), $20 \mathrm{~km}$ al S de Tulum, Cabrera C. 4349 (MEXU); Mpio. Felipe Carrillo Puerto: $19 \mathrm{~km}$ al NO de F. Carrillo Puerto sobre el camino a Vigía Chico, Cabrera C. 16977 (MEXU); Mpio. Felipe Carrillo Puerto: 3-6 km al SE del crucero Chumpon, Duran 623, 684 (MEXU); Mpio. Felipe Carrillo Puerto: Reserva de la Biosfera Soam Káan, desviación de la car. de Tulum a Felipe Carrillo Puerto a Vigia Chico, altitud 5 m, Duno de Stefano 1878 (CICY, MEXU); Isla Mujeres: Cancun, $11 \mathrm{~km}$ al N, camino del ejido R. Chaamunchic, Méndez 946 (MEXU); Mpio. José Ma. Morelos: 0.57 km del poblado Hno. Cruz, altitud 116 m, Álvarez 8368 (MEXU); Mpio. Lázaro Cárdenas: $10 \mathrm{~m}$ de la car. Cancun-Tulum, junto a la desviación al aeropuerto, Patricia Moreno 344 (MEXU); Mpio. Lázaro Cárdenas: $10 \mathrm{~km}$ al SO de Cancun, car. a Leona Vicario, Sousa 10852 (MEXU); Mpio. Lázaro Cárdenas: $8 \mathrm{~km}$ al N-NE de Pto. Morelos, en la car. a Cancun, Sousa 10865 (MEXU); Mpio. Lázaro Cárdenas: Kantuníl, rumbo a Cedral, altitud $12 \mathrm{~m}$, E. Ucán 2000 (XAL); Mpio. Othon P. Blanco: $10 \mathrm{~km}$ al S de San José, rumbo a Tomas Garrido, Cabrera C. 681 (MEXU); Mpio. Othon P. Blanco: en El Palmar a $7 \mathrm{~km}$ al S de Ucum, car. Chetumal-La Unión, Cabrera C. 1304, 4222 (MEXU); Mpio. Othon P. Blanco: $2 \mathrm{~km}$ al $\mathrm{E}$ de Calderitas, en Chetumal, en la orilla de la Bahía de Calderitas, Cabrera C. 1582 (MEXU); Mpio. Othon P. Blanco: $600 \mathrm{~m}$ del desvío hacia Chichan Ha, camino a Tomás Garrido, Durán 2770 (MEXU); Mpio. Othon P. Blanco: $3 \mathrm{~km}$ al S de Calderón, Méndez 427 (MEXU); Mpio. Othon P. Blanco: 9 km antes de X-Pujil llegando a Chetumal, altitud 100 m, Ucan 3697 (MEXU). BELICE, Belize, $20 \mathrm{~km}$ de Belice, por la car. Belice-Orange, altitud 10 m, Calzada 6933 (CICY); Gracie Rock 1.5-4 mi. S of mile 22 on western hwy., altitud $100 \mathrm{~m}$, Ronald Liesner 1456 (MEXU); La Democracia, 23 km al SO de Hattieville, Sousa 4171 (MEXU); $18 \mathrm{~km}$ al SO de Hattieville, en western hwy., altitud 90 m, Sousa 12131 (MEXU). Corozal, Can Lun road $3 \mathrm{~km} \mathrm{~W}$ of Calcutta, altitud 10 m, Michael J. Balick 2191 (MEXU); San Andrés, P. H. Gentle 1082 (DS, GH, MICH, MO). El Cayo, Benque Viejo, $7 \mathrm{~km}$ on Arenas Road, Elias Contreras 7046 (MO); Benque Viejo, S-SW, bordering Río Mopan, Contreras 7162 (MEXU); 41 mi. Section, Humming Bird hwy., Percy H. Gentle 8985 (IJ, MEXU, US); 38 mi. section Belize-El Cayo Road, Gentle 9275 (IJ, MICH, MO, TEX, US); cerca de Roaring Creek, T. P. Ramamoorthy 3681 (MEXU); 1 km al O de Austine, Sousa 4184 (MEXU); Cotton Tree Village, 9 km al E de Belmopan, altitud 50 m, Sousa 12072 (MEXU). Orange Walk, Duck Ridge, C. Leyland 428 (MEXU); $18 \mathrm{~km}$ al SE de Orange Walk, altitud $60 \mathrm{~m}$, Sousa
12052 (MEXU). Stann Creek, Sapon Road, Gentle 8119 (MO). Toledo, near border of Stann Creek along southern hwy. Thomas B. Croat 24188 (MEXU); Maya Mountains, canyon along Bladen Branch from Richardson Creek to Quebrada de Oro, altitud 100-200 m, Gerrit Davidse 32221 (MEXU); southern Maya Mountains, Bladen Nature Reserve, El Xux Canyon, ca. 1.8 airline km NW of the Ek Xux archeological site, altitud 320 m, Davidse 36166 (MEXU); Río Sarstoon (Belize-Guatemala border) mouth 5 mi. in, Dwyer 14862 (MEXU); Jacinto Creek, Río Grande, Gentle 4936 (IJ, MEXU, TEX); near San Antonio, in cohune ridge, Gentle 5482, 6649 (MO); betw. Punta Gorda and Paslow, in broken cohune ridge, Gentle 7806 (MO); SW Maya Mountains, Columbia River, Toledo Forest Reserve, trail between Gloria Camp and Edwards Camp to the S., Bruce K. Holst 4451 (MEXU). GUATEMALA, Alta Verapaz, Mpio. Panzós: along road to Hidrochulac and Cahobón from (N of) Tactic-El Estor road, altitud 650-750 m, W. D. Stevens et al. 25329 (MEXU); betw. Sachaj and Sacacac, on south side of Cerro Chinaja, altitud 150-180 m, Julian A. Steyermark 45149 (F); along Río Sebol, betw. Sebol and Carrizal, N of Sebol, altitud 200-300 m, Steyermark 45744 (A, F). El Peten, Tikal, Bajo de Santa Fé, Contreras 469 (F, MEXU); Dos Lagunas, bordering the airfield, Contreras 1554 (F, LL, MEXU); Dos Lagunas, about $10 \mathrm{~km}$ NW of the village, Contreras 1710 (F, LL, MEXU); Sto. Toribio, Contreras 2766 (F, LL, MO); Dolores betw. km 85-86 W of the Machaquila Road, Contreras 2936 (MEXU); Sayaxche, Laguna Petexbatun, alt. $300 \mathrm{~m}, \mathrm{~W}$ of Escarbadero, bordering the lake, Contreras 4155 (F, LL, MEXU); Santa Elena, km 7 of Remate Road, Contreras 6127 (F, MEXU); Lake Peten Itza, San Miguel 2 km, Contreras 7288 (F, MEXU); bordering Lake Peten Itza betw. San José and Chachaclun, Contreras 10351, 10362 (F, LL, MEXU); W of the Poptun road, about $2.5 \mathrm{~km}$ at the village, Cadenas on river bank, bordering Río Gracias clearing on Ramirito Rande, Contreras 19034 (F, MEXU); Tikal, Bajo de Santa Fe, near Aguada Pucte, Lundell 17023 (F, LL, MEXU); Tikal, Aguada Terminos, bordering Bajo Santa Fé, Lundell 17058 (F, LL, MEXU); Sayaxche bordering airfield, Lundell 17767 (LL, MEXU); $15 \mathrm{~km}$ entre Santa Elena y San Francisco, altitud 140 m, Antonio Molina R. 15641 (F); $28 \mathrm{~km}$ al S de Flores, altitud $160 \mathrm{~m}$, Sousa 4206 (MEXU); camino a El Remate a aprox. $10 \mathrm{~km}$ Parque Nal. de El Tikal, Rolando Tun Ortiz 315 (F); orillando camino para San Andrés a $10 \mathrm{~km}$, Santa Elena, Tun Ortiz 540 (F); Flores, entre Ixhu y el Aeropuerto, altitud 350 m, Véliz 7457 (BIGU, MEXU); La Libertad, sitio arqueológico La Joyanca, altitud 100 m, Véliz 8208 (BIGU, MEXU). 
Izabal, al lado del Río Dulce, 5 km NE del Puente Izabal, altitud 5 m, Douglas Kendig 6-5 (USCG); along the Río Dulce, downstream from the wide part called "El Golfete", from the hot springs about $3 \mathrm{~km}$, altitud $10 \mathrm{~m}$, M. Nee 47299 (MEXU); finca Murciélago, ca. $10 \mathrm{mi}$. E of El Estor on Lake Izabal, altitud $50 \mathrm{ft}$, Hugh Popenoe 58 (F); slopes WNW of (above) El Estor, along margin of open pit nickel mine, altitud $210 \mathrm{~m}$, Stevens 25264 (MEXU); La Mina de Eximibal, al E de El Estor, altitud $50 \mathrm{~m}$, Pedro Tenorio Lezama 14562 (MEXU); El Estor, Chichipate, altitud 300 m, M. Véliz 6549 (MEXU). Zacapa, above Teculutan, along Río Teculutan, altitud 250-275 m, Steyermark 42114 (F).

Discusión. La subespecie se caracteriza por sus largos tricomas en los organos vegetativos; estípulas persistentes; folíolos grandes; el lóbulo carinal del cáliz mucho más largo que los laterales y sus plántulas con los cotiledones hipogeos.

Lonchocarpus rugosus Benth. subsp. stipulaceus $M$. Sousa, subsp. nov. Tipo: México, Oaxaca: Cerro de Oro, Mpio. San Lucas Ojitlán, Distr. Tuxtepec, altitud 25 m, $M$. Sousa S., A. Delgado S. y A. Solís M. 7261 (holotipo, MEXU!). Figura 2.

Nombres comunes: Borrego (Veracruz); rosadillo (Oaxaca).

Arbor ad $15 \mathrm{~m}$ alta; surculi trichomatibus ad $2 \mathrm{~mm}$ longis. Folia 15-19-foliolata, foliolis 1.2-1.5(-2) cm latis, ellipticis vel lanceolatis; stipulae 10-19 mm longae, obovatae, apice mucronatae, basi 2-3(-4) mm, persistentes; calyx lobulo carinali centrali quam lateralibus leviter longiore vel fere aequanti; ovarium (5-)6-7-ovulatum. Legumen 2.2-3.8 cm latum, valvis laevigatis, marginibus constrictis. Plantula cotyledonibus epigaeis.

Árboles hasta $15 \mathrm{~m}$ de alto; tricomas de brotes vegetativos hasta $2 \mathrm{~mm}$ de largo. Hojas 15-19-folioladas; folíolos 1.2-1.5 (-2) cm de ancho, elípticos a lanceolados; estípulas 10-19 mm de largo, obovadas, ápice mucronado, la base 2-3 (-4) mm de ancho, persistentes; cáliz con el lóbulo carinal central ligeramente más largo que los laterales a casi tan largo como ellos; ovario (5-) 6-7-ovulado. Legumbre 2.2-3.8 cm de ancho, valvas lisas, márgenes constrictos. Plántulas con los cotiledones epígeos.

Distribución, hábitat y fenología. Esta subespecie nueva es endémica de la vertiente de la planicie costera del Golfo de México, en Veracruz y Oaxaca, en el área fitogeográfica mesoamericana de México. Prospera en selvas altas subperennifolias a perennifolias. En altitudes 10-250 (-400) $\mathrm{m}$. Florece de finales de agosto a principios de noviembre y fructifica de principios de diciembre a mediados de junio.

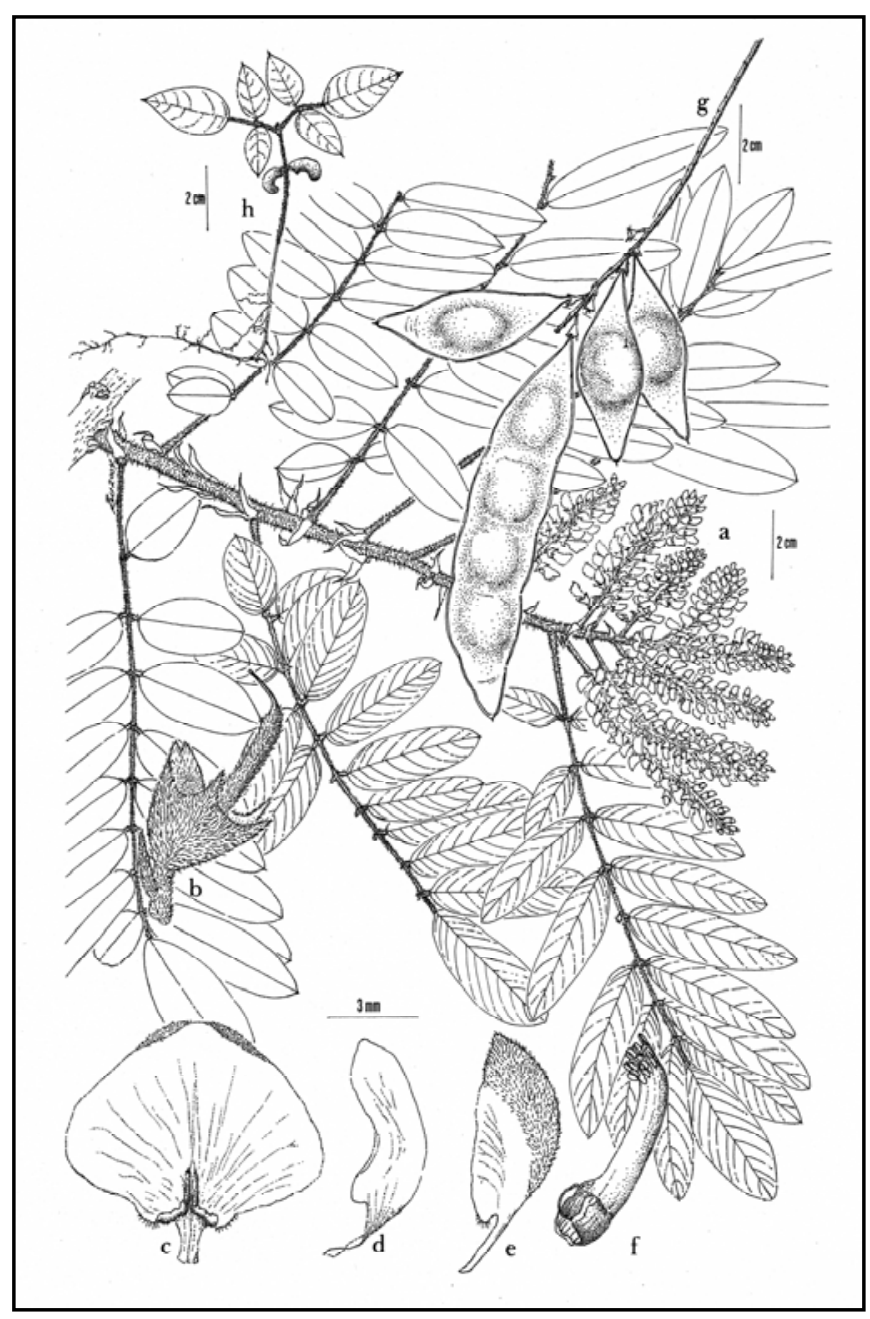

Figura 2. Lonchocarpus rugosus Benth. subsp. stipulaceus M. Sousa. A) Rama con hojas e inflorescencias. B) Cáliz y gineceo. C) Estandarte. D) Ala. E) Quilla. F) Tubo estaminal. Tomado de Sousa et al. 7982. G) Infrutescencia. Tomado de $R$. Torres 10945. H) Plántula, mostrando los cotiledones epigeos. Tomado de R. Torres 10945.

Material adicional revisado: MÉXICO, Oaxaca, Distr. Tuxtepec, Mpio. Acatlán de Pérez Figueroa: Aserradero 5 km al E de Acatlán de Pérez, altitud 200 m, M. Sousa S. 7982 (MEXU); Mpio. Acatlán de Pérez Figueroa: Tetela, altitud 100 m, Sousa 8842 (MEXU); Mpio. Acatlán de 
Sousa S., M.: Las subespecies de Lonchocarpus rugosus Benth. (Leguminosae, Papilionoideae: Millettieae)

Pérez Figueroa: $6 \mathrm{~km}$ al $\mathrm{S}$ de La Margarita, Vicente Camalote, entrando por el Amate al O de Tierra Blanca, Rafael Torres Colín 7783 (MEXU); Mpio. Ayotzintepec: 3 $\mathrm{km}$ al S de Reforma, camino a Ayotzintepec, altitud $100 \mathrm{~m}$, Esteban M. Martínez Salas 22215 (MEXU); Mpio. San Felipe Usila: potrero de los Hernández, en San Felipe Usila, altitud 145 m, J. Ismael Calzada 16962 (MEXU); Mpio. San José Chiltepec: Chiltepec, Sousa 4405a (MEXU); Mpio. San Juan Bautista Tuxtepec: Agua Escondida, Ejido Benito Juárez, Sebastopol, altitud 50 m, Brigada Dioscóreas 1799, 1927 (MEXU); Mpio. San Juan Bautista Tuxtepec: San Bartolo 2 km al NE de Tuxtepec, C. H. García Arizpe s. n. (MEXU); Mpio. San Juan Bautista Tuxtepec: altitud 100 m, G. Martínez-Calderón 1510 (INEGI, MEXU); Mpio. San Juan Bautista Tuxtepec: Loma del Chivo, a $13 \mathrm{~km}$ al SE de Tuxtepec, altitud 10 m, Sousa 10284 (MEXU); Mpio. San Lucas Ojitlan: de Loma Bonita a Loma Alta, por la car. para San Lucas Ojitlan, altitud 60 m, Calzada 14381 (MEXU); Mpio. San Lucas Ojitlán: Arroyo grande, Rafael Hernández Ortega 27 (MEXU); Mpio. San Lucas Ojitlán, 20 km al O de Paso Canoa, car. a Jalapa de Díaz o sea $3 \mathrm{~km}$ al O de San Lucas Ojitlán, altitud 100 m, Torres 9080 (MEXU); Mpio. Santa María Jacatepec: car. a Mauro Márquez a 3 km al S de La Reforma, altitud 40 m, Sousa 7274 (MEXU); Mpio. Temascal: Temascal, Rafael Hernández Magaña 737 (MEXU); Mpio. Temascal: cortina Presa Temascal, altitud 50 m, Sousa 1621, 13410 (MEXU); Mpio. Temascal: 2 km al E de Temascal, enfrente de la Compañía de Electricidad, Pedro Tenorio Lezama 19205 (MEXU); Mpio. Temascal: El Embarcadero, orilla E de la Presa Miguel Alemán, Temascal, Tenorio 19216 (MEXU); Mpio. Temascal: El Polvorín, $5 \mathrm{~km}$ de la cortina de la Presa de Temascal, camino a los Vertederos, altitud 187 m, Tenorio 19558 (MEXU); Mpio. Temascal: camino a la cortina de la Presa Miguel Alemán, Torres 10945 (MEXU). Veracruz, Mpio. Atoyac: $5 \mathrm{~km}$ al $\mathrm{S}$ de Caballo Blanco, altitud $350 \mathrm{~m}, R$. Acevedo R. 451 (MEXU); Mpio. San Andrés Tuxtla: El Salto de Eyipantla a $8 \mathrm{~km}$ del pueblo de Sihuapán, altitud 200 m, Calzada 1631 (MEXU); Mpio. San Andrés Tuxtla: en el Salto de Eyipantla, a 8 km de Sihuapán, altitud 250 m, Calzada 4242 (XAL); Mpio. Santiago Tuxtla: Sta. Rosa Hueyapan de Ocampo, Refugio Cedillo Trigos 385 (MEXU, XAL); Mpio. Santiago Tuxtla: Palmira, $24 \mathrm{~km}$ al S de Santiago Tuxtla y $1 \mathrm{~km}$ al N de Morillo, Sousa 4280 (MEXU).
Etimología. El nombre de la subespecie, hace énfasis en las estípulas, por su tamaño y persistencia.

Discusión. Subespecie muy característica por su larga pelosidad en los órganos vegetativos, sus enormes estípulas, persistente y plántulas con los cotiledones epigeos.

Agradecimientos. Para Fernando Chiang C. por su traducción al latín de la diagnosis; a Felipe Aguirre Ríos, por la elaboración de un banco de datos de las exsiccata. Para Gloria Andrade M. por organizar la información del manuscrito y recabar datos para su integración. A Ramiro Cruz por sus magníficas láminas botánicas. Por facilitar el material botánico a los curadores de los siguientes herbarios A, B, BIGU, BM, BR, CICY, COLO, DS, DUKE, EAP, ENCB, F, G, GH, IJ, INEGI, LL, MEXU, MICH, MO, NY, SMU, TEX, US, USCG, VT, XAL, quienes permitieron su consulta y estudio.

\section{Literatura Citada}

Hermann, F.J. 1948. Studies in Lonchocarpus and related genera, IV: The Lonchocarpus rugosus complex and additional Middle American species. J. Wash. Acad. Sc. 38(9): 310-312.

Holdridge, L.R. y L.J. Poveda A. 1975. Árboles de Costa Rica. Vol. 1. Palmas, otras monocotiledóneas arbóreas y árboles con hojas compuestas o lobuladas. Centro Científico Tropical, Costa Rica.

Pittier, H. 1917. The Middle American species of Lonchocarpus. Contr. U.S. Natl. Herb. 20: 37-93.

Sousa S., M. 1987. Lonchocarpus H.B.K. En: R. McVaugh. Leguminosae. En : W.R. Anderson (ed.) Flora NovoGaliciana 5: 555-577. Ann Arbor. Universiy of Michigan Press.

Witsberger, D., D. Current y E. Archer. 1982 [1983]. Árboles del Parque Deininger. Ministerio de Educación, San Salvador, p. 1-336.

Recibido para publicación el 30 de noviembre de 2007. 